\title{
Could fecal calprotectin enter mainstream use for diagnosing and monitoring inflammatory bowel disease?
}

\author{
Shu Chen Wei \\ Department of Internal Medicine, National Taiwan University Hospital and College of Medicine, Taipei, Taiwan
}

\section{Article: Accuracy of three different fecal calprotectin tests in the diagnosis of inflammatory bowel disease (Intest Res 2016;14:305-313)}

Calprotectin is a heterodimer of the calcium binding proteins S100A8 and S100A9, and is mainly present in neutrophils. ${ }^{1}$ Fecal calprotectin levels are correlated with the degree of intestinal inflammation, and have been found to increase when neutrophils migrate into the bowel lumen due to the inflammatory process. ${ }^{2}$ Therefore, fecal calprotectin could help discriminate between IBD and IBS, ${ }^{3}$ monitor treatment response and endoscopic disease activity, ${ }^{4,5}$ and predict relapse for IBD. ${ }^{6}$ Studies have also shown that fecal calprotectin levels are correlated with endoscopic severity scores ${ }^{5}$ and the extent of inflammation in Asian patients with UC. ${ }^{7}$ Coupled with the fact that it is a non-invasive and easily accessible test, the use of fecal calprotectin tests is expected to rise.

In this issue, Jang et al. ${ }^{8}$ compared three kinds of fecal calprotectin assay kits (Quantum Blue ${ }^{\circledR}$ from Bühlmann Laborotories, Basel, Switzerland; Eli ${ }^{\mathrm{TM}}$ from Phadia AB, Uppsala, Sweden; and RIDASCREEN ${ }^{\circledR}$ from R-Biopharm AG, Darmstadt, Germany) in the diagnosis of IBD. All three are point of care immunoassay tests. The authors compared the sensitivity, specificity, and positive and negative predictive values of these three kits in discriminating between IBD and IBS. For patients diagnosed with IBD, the kits were used to evaluate the correlation between fecal calprotectin and disease activity or location.

Received August 31, 2016. Revised September 4, 2016. Accepted September 5, 2016.

Correspondence to Shu Chen Wei, Department of Internal Medicine, National Taiwan University Hospital and College of Medicine, No.7 ChungShan South Road, Taipei 101, Taiwan. Tel: +886-2-23123456 ext. 65768, Fax: +886-2-23947927,E-mail: shuchenwei@ntu.edu.tw

Financial support: None. Conflict of interest: None.
The authors found that the overall accuracy for differentiating IBD from IBS or other types of colitis was $94 \%$ and $91 \%$ respectively for Quantum Blue ${ }^{\circledR}$ (cutoff, $50 \mu \mathrm{g} / \mathrm{g}$ ); 92\% and 89\% for EliA ${ }^{\mathrm{TM}}$ (cutoff, $50 \mu \mathrm{g} / \mathrm{g}$ ); and $82 \%$ and $76 \%$ for RIDASCREEN $^{\circledR}$ (cutoff, $50 \mu \mathrm{g} / \mathrm{g}$ ). The Quantum Blue ${ }^{\circledR}$ Calprotectin and $\mathrm{EliA}^{\mathrm{TM}}$ Calprotectin results were significantly correlated with the CDAI (Spearman's rank correlation coefficient $\mathrm{r}=0.66$ and 0.49 respectively) in patients with CD. EliA ${ }^{\mathrm{TM}} \mathrm{Cal}-$ protectin was significantly correlated with the Mayo score $(\mathrm{r}=0.70)$ in UC patients. Thus, the authors concluded that fecal calprotectin levels were useful in identifying IBD. Overall, these three fecal calprotectin kits were comparable in accuracy.

Although this was a small-scale study, it provided practical and useful information on these three kits. This can help guide our daily clinical practice in terms of choosing an appropriate kit. Their results clearly demonstrated that all three fecal calprotectin kits were superior to CRP in distinguishing IBD from IBS and other types of colitis. In addition, the overall accuracies of these three kits were comparable. However, although there was good correlation between the different kits, it is inappropriate to directly compare the absolute calprotectin levels between the kits. Instead, the same kit should be used for follow-up comparisons. At present, cost appears to be the main factor affecting the choice of kit. The authors noted that the prices range from 20 to 33 US dollars per test, but also explained that the final cost might vary according to the number of tests submitted. When the availability and cost issues have been resolved, we can expect that fecal calprotectin will enter mainstream use for the diagnosis and monitoring of IBD.

\footnotetext{
๑ Copyright 2016. Korean Association for the Study of Intestinal Diseases. All rights reserved.

This is an Open Access article distributed under the terms of the Creative Commons Attribution Non-Commercial License (http://creativecommons.org/licenses/by-nc/4.0)

which permits unrestricted non-commercial use, distribution, and reproduction in any medium, provided the original work is properly cited.
} 


\section{REFERENCES}

1. Hsu K, Champaiboon C, Guenther BD, et al. Anti-infective protective properties of S100 calgranulins. Antiinflamm Antiallergy Agents Med Chem 2009;8:290-305.

2. Tibble J, Teahon K, Thjodleifsson B, et al. A simple method for assessing intestinal inflammation in Crohn's disease. Gut 2000;47:506-513.

3. Chang MH, Chou JW, Chen SM, et al. Faecal calprotectin as a novel biomarker for differentiating between inflammatory bowel disease and irritable bowel syndrome. Mol Med Rep 2014;10:522-526.

4. D'Haens G, Ferrante M, Vermeire S, et al. Fecal calprotectin is a surrogate marker for endoscopic lesions in inflammatory bowel disease. Inflamm Bowel Dis 2012;18:2218-2224.
5. Lin WC, Wong JM, Tung CC, et al. Fecal calprotectin correlated with endoscopic remission for Asian inflammatory bowel disease patients. World J Gastroenterol 2015;21:13566-13573.

6. Wright EK, Kamm MA, De Cruz P, et al. Measurement of fecal calprotectin improves monitoring and detection of recurrence of Crohn's disease after surgery. Gastroenterology 2015;148:938-947.e1.

7. Kawashima K, Ishihara S, Yuki T, et al. Fecal calprotectin level correlated with both endoscopic severity and disease extent in ulcerative colitis. BMC Gastroenterol 2016;16:47.

8. Jang HW, Kim HS, Park SJ, et al. Accuracy of three different fecal calprotectin tests in the diagnosis of inflammatory bowel disease. Intest Res 2016;14:305-313. 